\title{
Darf der Staat von Migrant*innen fordern, sich zu integrieren?
}

\author{
Tobias Gutmann
}

Eingegangen: 22. Juni 2021 / Angenommen: 1. September 2021 / Online publiziert: 27. Oktober 2021

(C) Der/die Autor(en) 2021

Zusammenfassung In diesem Aufsatz gehe ich der Frage nach, ob der Staat von Migrant*innen fordern darf, sich zu integrieren. Nach einer Erläuterung des Begriffs „Integration“ und der verschiedenen Dimensionen der Integration werde ich ein Argument vorstellen, das zeigt, dass sich auf Grundlage des politischen Liberalismus eine staatliche Forderung nach politischer Partizipation von Migrant*innen begründen lässt. Diese Forderung beruht auf Gerechtigkeits- sowie demokratietheoretischen Überlegungen. Weil die politische Partizipation zugleich eine gewisse strukturelle, soziale und identifikative Integration der Migrant*innen voraussetzt, erstreckt sich die Forderung auch auf diese Voraussetzungen. Da sich die entsprechende Forderung gleichermaßen an Migrant*innen als auch an Einheimische richtet, entgeht die hier vorgestellte Argumentation dem Vorwurf, auf einem bloß partikularen Interesse der einheimischen Bevölkerung zu beruhen, dass sich die Migrant*innen der bzw. einer nationalen Kultur anpassen mögen. Zuletzt diskutiere ich einen Einwand, der sich gegen eine der Prämissen meiner Argumentation richtet. Dieser lautet, dass ein liberaler Staat keinen Einfluss auf die Haltungen und Überzeugungen seiner Bürger*innen nehmen darf. Ich verteidige die Behauptung, dass es auch im liberalen Staat legitim ist, die Bürger*innen zur Demokratie zu erziehen.

Schlüsselwörter Integration · Migration · Politischer Liberalismus · Demokratie · Erziehung zur Demokratie

\footnotetext{
Tobias Gutmann $(\bowtie)$

Zentrum für Lehrerbildung, Technische Universität Chemnitz, Straße der

Nationen 12, 09111 Chemnitz, Deutschland

E-Mail: tobias.gutmann@zlb.tu-chemnitz.de
} 


\section{Can the state demand that migrants integrate?}

Abstract In this paper, I will explore the question of whether the state may require migrants to integrate. I start with an explanation of the term "integration" and differentiate between several dimensions of integration. Thereafter I will present an argument on the basis of political liberalism that shows that the state may demand political participation from migrants. This demand is based on considerations of justice and democratic theory. Because political participation presupposes a certain degree of structural, social and identificational integration of migrants, the demand also extends to these preconditions. Since such a demand is directed equally at migrants and natives, the argumentation presented here escapes the accusation of being based on a merely particular interest of the native population that migrants should adapt to a national culture. Finally, I discuss an objection that is directed against one of the premises of my argumentation. This objection is that a liberal state must not influence the attitudes and beliefs of its citizens. I defend the claim that even in a liberal state it is legitimate to educate citizens for democracy.

Keywords Integration $\cdot$ Migration $\cdot$ Political liberalism $\cdot$ Democracy $\cdot$ Education for democracy

\section{Einleitung}

Mit Fragen der Einwanderung beschäftigt sich die politische Philosophie schon seit geraumer Zeit. Dabei gibt es zwei große Diskussionsstränge: Im ersten steht vor allem die Frage nach der Legitimität von Zuwanderungsbeschränkungen im Vordergrund, also die Frage danach, ob und wann es erlaubt sein kann, Migrant*innen am Überschreiten von Staatsgrenzen zu hindern. ${ }^{1}$ Der andere Diskussionsstrang befasst sich mit Fragen im Zusammenhang mit der Integration der Migrant*innen. Zu diesen Fragen gehören etwa die folgenden: Was ist gelingende Integration? Welche Rechte sollen die neu ankommenden Menschen haben? Gehen mit diesen Rechten auch Pflichten einher? Welche Pflichten haben Staaten gegenüber den Einwandernden? Besteht auch für die schon im Land lebende Bevölkerung eine Pflicht, einen Beitrag zu leisten zur gelingenden Integration? Worin genau gründen diese Rechte und Pflichten? ${ }^{2}$

Diese beiden Diskussionsstränge betreffen nicht vollkommen voneinander unabhängige Fragen, wie die folgenden Überlegungen zeigen sollen: Erstens zeigt ein Blick in die Literatur zur Frage nach der Legitimität von Zuwanderungsbeschränkungen, dass viele Befürworter*innen eines Rechts auf Einwanderung dieses nicht als absolutes Recht verstehen, sondern als eines, das, zumindest in einigen Fällen, Einschränkungen erfahren darf. Solche Einschränkungen können sich aus Komplikationen ergeben, welche Migration zumindest potentiell auch mit sich bringt. So ist etwa Ladwig (2012: 80) der Ansicht, dass Einwanderungsbeschränkungen erlaubt

1 So etwa in Wellman und Cole (2011), Carens (1987), Cassee (2016) und Dietrich (2017).

2 Siehe zu solchen Fragen Carens (2015: 62-87) und Miller (2016: 130-150). 
sind, um eine gerechte Ordnung innerhalb eines Staates zu bewahren. Carens (1987: 259) zufolge darf die Einwanderung beschränkt werden, um einen Zusammenbruch der öffentlichen Ordnung zu verhindern und laut Cassee (2016: 277) kann das Recht auf Bewegungsfreiheit eingeschränkt werden, wenn ein plötzlicher kultureller Bruch droht, der nicht auf andere Weise verhindert werden kann. Insofern die Integration von Migrant*innen einen Einfluss auf diese potentiellen Komplikationen hat, können Überlegungen zur Legitimität der Forderung nach Integration also auch zu einem genaueren Verständnis des Rechts auf Einwanderung beitragen.

Zweitens kann die Beschäftigung mit den die Integration betreffenden Fragen von moralstrategischer Bedeutung sein: Wenn man davon ausgeht, dass die Bereitschaft des Staates und der Bevölkerung, weitere Migrant*innen aufzunehmen, gröBer sein wird, wenn deren Integration gelingt, dann könnten die Bemühungen um ein adäquates Verständnis von gelingender Integration - insofern dieses Verständnis weitere Verbreitung findet und sich die Politik daran orientiert - dazu beitragen, die Bereitschaft zur weiteren Aufnahme von Migrant*innen zu erhöhen (Neuhäuser 2015: 399). Ein Hinweis darauf, dass ein solcher Zusammenhang tatsächlich bestehen könnte, besteht darin, dass das Thema „Integration“ in der öffentlichen Diskussion eine große Rolle spielt. So finden sich in den vergangenen Jahren in deutschsprachigen Medien sehr viele Beiträge, in denen die ge- oder misslungene Integration von Migrant*innen konstatiert, kritisiert und diskutiert wird. Ein wiederkehrendes Thema dabei ist etwa die Frage nach einer „Leitkultur“, an der sich die Integration von Migrant*innen orientieren solle (Lammert 2006; de Maizière 2017). Oftmals wird in derartigen Debatten pauschalisierend davon ausgegangen, dass der Integrationsbedarf allein bei Migrant*innen vorhanden oder doch bei ihnen sehr viel größer sei. Diese Pauschalurteile sind sicherlich falsch; und so stellt sich neben der Frage, ob der Staat von Migrant*innen fordern darf, sich zu integrieren, auch die Frage, ob der Staat dies von Einheimischen verlangen darf. Nichtsdestotrotz rechtfertigen die aufgezeigten Verbindungen zur Frage nach der Zulässigkeit von Zuwanderungsbeschränkungen und den moralstrategischen Überlegungen eine Fokussierung auf die titelgebende Frage, die hier auch in der Hoffnung beantwortet werden soll, zu zeigen, dass die oftmals hysterisch geführte öffentliche Debatte durch eine sachliche ersetzt werden kann.

In diesem Beitrag will ich darum der Frage nachgehen, ob sich staatliche Forderungen danach, dass Migrant*innen sich integrieren sollen, begründen lassen. Dazu werde ich zuerst den Begriff ,Integration“ erläutern und dann zeigen, dass sich auf Grundlage des politischen Liberalismus eine Forderung zur politischen Partizipation begründen lässt, die plausiblerweise auch eine gewisse strukturelle, kulturelle, soziale und identifikative Integration der Migrant*innen voraussetzt. Diese Forderung ergibt sich aus Überlegungen zur Gerechtigkeit sowie demokratietheoretischen Überlegungen. Weil sich eine entsprechende Forderung gleichermaßen an Einheimische richtet, entgeht die hier vorgestellte Begründung der Forderung nach Integration von Migrant*innen dem Vorwurf, auf einem bloß partikularen Interesse der einheimischen Bevölkerung zu beruhen, dass sich die Migrant*innen der bzw. einer nationalen Kultur anpassen mögen. ${ }^{3}$

\footnotetext{
3 Dass die Integration allzu oft als derartige Aufforderung verstanden wird, kritisiert z.B. Özmen (2015).
} 


\section{Zum Begriff „Integration“}

Der Begriff „Integration“ stammt aus der Soziologie. In der soziologischen Literatur findet sich zwar keine einheitliche Bestimmung dieses Begriffs (vgl. Friedrichs und Jagodzinski 2008: 94), weithin geteilt wird aber das Grundverständnis, dem zufolge „unter Integration - ganz allgemein - [...] der Zusammenhalt von Teilen in einem ,systemischen' Ganzen verstanden [wird], gleichgültig zunächst, worauf dieser Zusammenhalt beruht“, wobei die Teile „ein nicht wegzudenkender [...] Bestandteil des Ganzen sein“ müssen, das in seiner Umgebung ,als ,System“ identifizierbar“ wird. (Esser 2001: 1). Das Objekt der soziologischen Integrationsforschung sind dementsprechend oft ganze Gesellschaften, manchmal mehr oder weniger konkrete wie die deutsche Gesellschaft, manchmal aber auch eher vage wie etwa ,die moderne Gesellschaft“. Eine zentrale Frage solcher Untersuchungen lautet also: „Was hält die Gesellschaft zusammen?“ - so ein Titel, den in kleinen Variationen einige einschlägige Sammelbände tragen (Teufel 1996; Heitmeyer 1997a; Köppl 2013). In der Soziologie wird ein breites Spektrum von möglichen Faktoren diskutiert, die für den Zusammenhalt verantwortlich sind bzw. zum Zusammenhalt beitragen. Zu diesen Faktoren zählen unter anderem die Verwandtschaft, die Religion, die Arbeitsteilung, der Markt, die Nation, das Militär, die Verwaltung, die Sprache, das Recht, die Politik, die Kultur oder die Bürgergemeinschaft (Münch 1997). Mit der Frage nach einem einigenden Band von Gesellschaften beschäftigen sich mehr oder weniger explizit schon Klassiker der Philosophie und der Soziologie wie Hobbes, Rousseau, Burke, Hegel, Durkheim und Weber (Bedorf und Herrmann 2016). Dabei ist die Frage stets aktuell geblieben: Sie stellt sich vor dem Hintergrund der Diskussion der Legitimität politischer Herrschaft, erfährt neue Beachtung durch das Entstehen der Nationalstaaten vor allem im 19. Jahrhundert, und verschärft sich, zumindest nach Ansicht einiger Autor*innen, mit Entwicklungen wie etwa der Ausweitung kapitalistischer Denk- und Handlungsweisen, der Globalisierung, dem Entstehen demokratischer, pluralistischer, individualistischer Gesellschaften und dem Anwachsen der globalen Migration (Münch 1997; Heitmeyer 1997b). Schon dieser kurze Überblick macht deutlich, dass sich die Frage danach, was eine Gesellschaft zusammenhält, keinesfalls ausschließlich im Kontext der Ankunft von Migrant*innen stellt, wie etwa auch Heitmeyer (1997c: 9f.) festhält. Das wissenschaftliche Interesse an Fragen der Integration entsteht vielmehr allein schon deshalb, weil ,,mit der Ausprägung des Differenzierungsgrades einer Gesellschaft auch die Notwendigkeit erhöhter sozialer Integration erwartet wird“ (Heitmeyer 1997b: 25). Nur leicht verkürzt gesagt stellt sich demnach die Frage, was eine Gesellschaft zusammenhält, sobald man anerkennt, dass (moderne) Gesellschaften kein homogenes Ganzes sind, sondern sich zusammensetzen aus Mitgliedern, die verschiedenen Glaubensgemeinschaften, Schichten oder Kulturen usf. angehören und divergierende Interessen, Bedürfnisse, politische Ansichten usf. haben. Die Frage nach der Integration von Migrant*innen stellt also bloß einen Aspekt der allgemeineren Frage dar, was Gesellschaften zusammenhält.

In der Soziologie wird unterschieden zwischen ,Sozial-“ und „Systemintegration“. Die Systemintegration bezeichnet dabei die Integration, „die sich unabhängig von den speziellen Motiven und Beziehungen der individuellen Akteure und oft sogar auch gegen ihre Absichten und Interessen, sozusagen anonym und hinter ih- 
rem Rücken, ergibt und durchsetzt, während die soziale Integration unmittelbar mit den Motiven, Orientierungen und - insbesondere - den Beziehungen der Akteure zu tun hat" (Esser 2000: 270). Die Systemintegration kann sich z.B. durch das Eingebundensein in Marktprozesse ergeben und dadurch, dass man von Gesetzen betroffen ist; die Sozialintegration etwa durch den Erwerb einer neuen Sprache oder politische Partizipation. Darüber hinaus werden verschiedene Dimensionen der Integration unterschieden. Die strukturelle Integration bezieht sich auf die Mitgliedschaft in bzw. Teilhabe an zentralen Institutionen einer Gesellschaft; dazu zählen etwa das Bildungs- und Ausbildungssystem, der Arbeits- und Wohnungsmarkt, die sozialen Sicherungssysteme und die politische Gemeinschaft (Heckmann 2015: 72). Ein zentraler Aspekt der strukturellen Integration, nämlich die Teilhabe am politischen Geschehen, kann mit dem Begriff politische Integration bezeichnet werden (Habermas 1992: 644). Die kulturelle Integration bezieht sich auf den Erwerb von Kompetenzen, die für die Kommunikation und die Arbeit in einer Gesellschaft notwendig sind, und auf die Annahme von Werten, Normen, Einstellungen und Verhaltensweisen (Heckmann 2015: 159). Die soziale Integration bezieht sich auf das Bestehen bzw. die Herausbildung persönlicher Beziehungen mit anderen Mitgliedern der Gesellschaft (Heckmann 2015: 73) Die identifikative Integration bezeichnet die Identifizierung mit einer Gesellschaft oder einer Gruppe, die einhergeht mit einem Gefühl der Verantwortlichkeit bzw. Loyalität gegenüber dieser Gesellschaft oder Gruppe (Heckmann 2015: 194).

Oft wird der Begriff „Integration“ abgegrenzt vom Begriff „Assimilation“. Dies geschieht, insofern der Begriff „Assimilation“ verstanden wird als Ausdruck des Wunsches, kulturell homogene Staaten zu schaffen einhergehend mit ,gewaltsamen Versuchen, die Minderheiten in Konformität zur Mehrheit zu zwingen“ (Heckmann 2015: 75). Der Begriff beschreibt dann die einseitig an die Migrant*innen gerichtete Forderung, sich der Kultur eines Landes anzupassen bzw. diese zu übernehmen, wogegen der Begriff „Integration“ ein beiderseitiges Aufeinander-Zugehen und einen interkulturellen Austausch bezeichnet (Modood 2007: 46-51). Allerdings gibt es in der aktuellen Debatte auch Stimmen, denen zufolge der Begriff „Integration“ auf den gleichen Voraussetzungen beruhe wie der Begriff „Assimilation“ und damit die gleiche Forderung impliziere, nämlich die einseitige Übernahme der Kultur vonseiten der Migrant*innen (Hess und Moser 2009: $11 \mathrm{ff}$ ). Zugleich findet auch der Begriff „Assimilation“ in wissenschaftlichen Publikationen weiter Verwendung, z.B. zur Beschreibung der gegenseitigen Beeinflussung von Einwanderern und der einheimischen Bevölkerung (Heckmann 2015: 75-78). Oft drücken sich in diesen Uneinigkeiten über die angemessene Bezeichnung schon jeweils moralische Urteile darüber aus, welche Art von Anpassung vom wem gerechtfertigterweise gefordert werden darf.

Ich werde im Folgenden den heute gebräuchlicheren Begriff „Integration“ verwenden und diesen verstehen als Bezeichnung jener Prozesse und Maßnahmen, die Migrant*innen die Teilnahme bzw. Teilhabe am gesellschaftlichen Leben ermöglichen bzw. diese Teilnahme und Teilhabe befördern. Berücksichtigt werden sollen dabei die oben genannten Dimensionen der Integration, und so gehören zu diesen Prozessen und Maßnahmen etwa die Gewährung des Wahlrechts, die Migrant*innen die politische Partizipation ermöglicht, oder auch der Erwerb der Sprache bzw. einer 
der Sprachen des Aufnahmelandes, der die Kommunikation zwischen Migrant*innen und Einheimischen ermöglicht. Diese Prozesse und Maßnahmen können vom Aufnahmeland ausgehen - das ist der Fall bei der Gewährung des Wahlrechts; sie können von den Migrant*innen ausgehen - so ist das im Fall des Spracherwerbs; und sie können von den Einheimischen ausgehen - ein Beispiel dafür wäre eine privat organisierte Hilfe für neu ankommende Migrant*innen. Die in diesem Aufsatz im Mittelpunkt stehende Frage lautet demnach, ob der Staat ein Recht hat, von Migrant*innen zu fordern, bestimmte Handlungen auszuführen bzw. Einstellungen zu übernehmen, welche die Integration befördern.

\section{Die Forderung nach Integration}

Vor allem in der öffentlichen Debatte sind Forderungen danach, dass sich Migrant*innen einer bestimmten Kultur anpassen sollen, sehr präsent. In Deutschland sind hier vor allem die Versuche zu nennen, eine sogenannte ,deutsche Leitkultur“ zu beschreiben, an die sich Migrant*innen anzupassen hätten. ${ }^{4}$ Als prominenter Befürworter einer derartigen Forderung darf neben Friedrich Merz auch der ehemalige Bundesminister des Inneren, Thomas de Maizière gelten. Letzterem zufolge ist die Leitkultur ,über die Sprache, Verfassung und Achtung der Grundrechte“ hinaus das, „was uns [d.i. die Staatsbürgerinnen und Staatsbürger Deutschlands] im Innersten zusammenhält, was uns ausmacht und was uns von anderen unterscheidet“. Zur näheren Bestimmung zählt er einige ,Lebensgewohnheiten [auf], die es wert sind, erhalten zu werden“, unter anderem: ,Wir sagen unseren Namen. Wir geben uns zur Begrüßung die Hand. Bei Demonstrationen haben wir ein Vermummungsverbot. [...] Wir sehen Bildung und Erziehung als Wert und nicht allein als Instrument. [...] In unserem Land ist Religion Kitt und nicht Keil der Gesellschaft. [...] Für uns sind Respekt und Toleranz wichtig. Wir akzeptieren unterschiedliche Lebensformen und wer dies ablehnt, stellt sich außerhalb eines großen Konsenses. [...] Wir sind aufgeklärte Patrioten. [...] Wir haben ein gemeinsames kollektives Gedächtnis für Orte und Erinnerungen“. De Maizière zufolge wird „,die Integration kaum gelingen“ bei jenen, die ,eine solche Leitkultur weder kennen, vielleicht nicht kennen wollen oder gar ablehnen [...]“ (de Maizière 2017) - folglich sollen Migrant*innen diese Leitkultur übernehmen.

Worin kann eine solche Forderung nach Übernahme der Lebensgewohnheiten der einheimischen Bevölkerung gründen? Auf den ersten Blick bietet es sich an, für diese Forderung auf Grundlage des Kommunitarismus zu argumentieren. Kommunitaristen betonen, dass Menschen in erster Linie soziale Wesen sind, deren Identität und Selbstverständnis sich aus der kulturellen Zugehörigkeit und geteilten Traditionen speise. Aus diesem Grund seien eine geteilte Kultur und geteilte Traditionen

\footnotetext{
4 Der Begriff „Leitkultur“ wurde geprägt von Bassam Tibi, dem es dabei allerdings nicht um eine deutsche Leitkultur geht, sondern um eine europäische, die er versteht als ein „demokratischer, laizistischer sowie an der zivilisatorischen Identität Europas orientierter Wertekonsens“ (Tibi 2001). Einen kurzen Überblick über die nachfolgenden Aneignungen dieses Begriffs bieten Özmen (2015: 355-357) und Stein (2008: 4042).
} 
die Voraussetzungen dafür, zu wissen, was einem wichtig ist und damit auch die Grundlage autonomer Entscheidungen. ${ }^{5}$ Es ist kein großer Schritt von dieser Idee hin dazu, dass darum diejenigen, die Teil dieser Kultur und Traditionen sind, ein Recht darauf haben, zu bestimmen, was mit dieser Kultur und diesen Traditionen passieren soll. Genau diese Idee drückt etwa Walzer aus, wenn er schreibt, dass „Einreise- und Zuwanderungsbeschränkungen [...] den Zweck [haben], Freiheit und Wohlfahrt sowie Politik und Kultur einer Gruppe von Menschen zu bewahren, die sich einander und einem gemeinsamen Leben verpflichtet fühlen“(Walzer 2006: 76). Zwar argumentiert Walzer an dieser Stelle auf Grundlage des Wertes der gemeinsamen Kultur dafür, dass Einwanderungsbeschränkungen erlaubt sein können, aber es ließe sich auf derselben Grundlage auch zugunsten einer Forderung zur kulturellen Integration argumentieren. Dem Verlust oder der Veränderung der Kultur könnte auf beide Weisen entgegenwirkt werden. Dieses Recht, die geteilte Kultur zu bewahren, ist für Walzer ein kollektives Recht, das die Bürger*innen eines Staates haben: „Wir, die wir bereits Mitglieder sind, nehmen die Auswahl vor [darüber, wer aufgenommen werden soll und nach welchen Kriterien dies geschehen soll], und zwar gemäß unseres Verständnisses davon, was Mitgliedschaft in unserer Gemeinschaft bedeutet und welche Art von Gemeinschaft wir zu haben wünschen" (Walzer 2006: 66). In dieser Hinsicht gleichen Staaten laut Walzer Vereinen, die ihre Mitglieder aussuchen dürfen (Walzer 2006: 76f). Demzufolge dürfen also die Bürger*innen eines Staates bestimmen, wer im Staat aufgenommen wird - und obwohl auch an dieser Stelle nicht explizit die Rede davon ist, könnte auf diese Weise ebenso eine Forderung nach kultureller Integration begründet werden: wer aufgenommen werden will, muss sich der Gemeinschaft kulturell anpassen.

Die kommunitaristische Argumentation, zumindest in der Version von Walzer, sieht sich allerdings mit einigen Einwänden konfrontiert: Zum einen scheint er davon ausgehen zu müssen, dass es eine einheitliche Nationalkultur gibt, deren Grenzen mit den Grenzen des Nationalstaates zusammenfallen (Cassee 2016: 99). Zweitens ist nicht klar, ob man nicht mit der gleichen Überlegung zum Schluss gelangen kann, dass auch solcher Wandel unterbunden werden dürfte, der seinen Ausgang innerhalb der genannten Kultur nimmt, was einer dramatischen Einschränkung der Selbstbestimmungsrechte der Bürger*innen dieses Staates gleichkommen würde (Freiman und Hidalgo 2016: 7). Ich will diese Einwände und die möglichen Erwiderungen vonseiten des Kommunitarismus an dieser Stelle nicht weiterverfolgen, sondern stattdessen der Frage nachgehen, ob man die Forderung des Staates an die Migrant*innen, sich zu integrieren, auch auf Grundlage einer konkurrierenden Grundposition der politischen Philosophie begründen kann, nämlich dem Liberalismus.

Der Liberalismus kann angesehen werden als eine Reaktion auf das Scheitern philosophischer Versuche, die Frage nach dem guten Leben zu beantworten, sowie als Reaktion auf den Pluralismus, also die Tatsache, dass die Menschen auch innerhalb einer Gesellschaft z.B. unterschiedlichen Religionen angehören, unterschiedliche Lebensziele verfolgen und darüber uneins sind, wie ein gelungenes Leben aussieht (Larmore 1993: 131-136). An die Stelle einer für alle verbindlichen Lebensweise

\footnotetext{
5 Vgl. etwa die Erläuterungen zum Kommunitarismus in Kymlicka (1990: 207-237) und Gutmann (1993).
} 
setzt der Liberalismus das Prinzip der Achtung persönlicher Freiheiten. Zu diesen Freiheiten gehört auch die Freiheit, selbstbestimmt zu entscheiden, wie man leben will. Die Forderung, eine bestimmte Kultur zu übernehmen, widerspricht dieser Freiheit und ist damit aus liberaler Sicht nicht zu rechtfertigen. Ganz in diesem Sinne hält Habermas (2002: 13) fest: „In einem demokratischen Verfassungsstaat darf auch die Mehrheit den Minderheiten die eigene kulturelle Lebensform - soweit diese von der gemeinsamen politischen Kultur abweicht - nicht als sogenannte Leitkultur vorschreiben“". Offensichtlich spricht sich Habermas damit gegen eine Pflicht zur kulturellen Integration aus, die Kommunitaristen wie Walzer wohl gutheißen würden.

Der Vorzug, sich bei der Frage nach der Begründbarkeit einer Forderung nach Integration an Migrant*innen auf den Liberalismus zu berufen, besteht darin, dass dieser offensiv die Selbstbestimmungsrechte betont, an die immer wieder auch diejenigen appellieren, die sich gegen Forderungen wie die zu Beginn dieses Abschnitts zitierten wenden. So beruft sich etwa Özmen explizit auf die gerade zitierte Behauptung von Habermas, um gegen die Forderungen nach Anpassung der Migrant*innen an eine (mehr oder weniger geteilte) Kultur in einem Land zu argumentieren (Özmen 2015: $357 \mathrm{f}$.). Allerdings lässt sie in ihrem Zitat den Teil aus, den Habermas in Gedankenstriche gesetzt hat. Habermas behauptet, dass in einem demokratischen Verfassungsstaat die Mehrheitskultur die eigene kulturelle Lebensform nicht als Leitkultur vorschreiben darf, ,,soweit diese [die kulturelle Lebensform der Mehrheit] von der gemeinsamen politischen Kultur abweicht". Demnach betrifft das liberale Verbot, Migrant*innen vorzuschreiben, eine bestimmte Kultur zu übernehmen, Habermas zufolge nicht diejenigen Teile der Kultur, die zur gemeinsamen politischen Kultur gehören.

Aber lässt sich auf liberaler Grundlage auch zugunsten der Forderung der Übernahme einer bestimmten politischen Kultur argumentieren? Habermas lässt in seinem eben genannten Zitat zumindest die Möglichkeit offen, indem er implizit auf die Notwendigkeit einer gemeinsamen politischen Kultur hinweist. An anderer Stelle weist er auch explizit auf diese Notwendigkeit hin: „Die Identität des [demokratisch verfassten] politischen Gemeinwesens, die auch durch Immigration nicht angetastet werden darf, hängt primär an den in der politischen Kultur verankerten Rechtsprinzipien und nicht an einer besonderen ethnisch-kulturellen Lebensform im ganzen. Demnach muss von Einwanderern nur die Bereitschaft erwartet werden, dass sie sich auf die politische Kultur ihrer neuen Heimat einlassen, ohne deshalb die kulturelle Lebensform ihrer Herkunft aufgeben zu müssen“ (Habermas 1992: 658f). Auch im Rahmen des politischen Liberalismus kann demnach eine Forderung nach Integration legitim sein, eben die Forderung nach politischer Integration. Allerdings stellen sich hier zwei Fragen: Zum einen die Frage, wie man diese Forderung nach politischer Integration begründen kann, und zum anderen die Frage, was genau alles dazugehört zum, wie Habermas es formuliert, „Sich-einlassen“ auf die politische Kultur eines Landes. 


\section{Eine Begründung der Forderung nach politischer Integration}

In der Literatur ist man sich weitgehend darüber einig, dass Staaten ${ }^{6}$ die Pflicht haben, den längerfristig sich im Land aufhaltenden Migrant*innen das Recht der politischen Partizipation zuzugestehen. ${ }^{7}$ Begründet wird diese Pflicht unter Verweis auf das demokratische Ideal, dem zufolge diejenigen, die den politischen Entscheidungen eines Staates unterworfen sind, auch an der politischen Entscheidungsfindung beteiligt sein sollen. ${ }^{8}$ Da das Recht auf politische Partizipation in den meisten Fällen an die Staatsbürgerschaft geknüpft ist, lautet die Forderung, dass Staaten die Migrant*innen als Staatsbürgerinnen anerkennen sollen. Dieses Argument spricht also zuerst einmal zugunsten einer Forderung, die sich an den Staat wendet: Er soll dafür sorgen, dass Migrant*innen das Recht haben, politisch zu partizipieren. Kann man auf der gleichen Grundlage auch für eine Forderung argumentieren, die sich an die Migrant*innen richtet?

Ein Ausgangspunkt für eine derartige Begründung ist das Konzept der Staatsbürgerschaft in einem demokratischen Staat. Die Staatsbürgerschaft in einer Demokratie kann man verstehen als ein Bündel von Rechten - aber auch als ein Bündel an Pflichten. Als Bürger*in eines demokratischen Staates hat man nicht nur gewisse Rechte - etwa das aktive und passive Wahlrecht -, sondern auch bestimmte Pflichten. Bei manchen dieser Pflichten handelt es sich um sanktionsbewährte Rechtspflichten, d.h. ein Verstoß gegen diese Pflichten wird auch tatsächlich bestraft. So besteht etwa in den fünf EU-Ländern Belgien, Griechenland, Italien, Luxemburg und Zypern eine Wahlpflicht. In Belgien und Luxemburg können Geldstrafen verhängt werden gegen diejenigen, die sich nicht an den Wahlen beteiligen, in Griechenland kann man dafür sogar mit einer Haftstrafe von bis zu einem Jahr büßen. ${ }^{9}$ Eine weitere oft sanktionsbewährte Pflicht von Staatsbürgern (und zum Teil auch für Staatsbürgerinnen) ist die Wehrpflicht. So müssen etwa in Israel, Norwegen und Schweden die Bürger*innen damit rechnen, zum Wehrdienst eingezogen zu werden. Neben diesen rechtlichen bestehen aber auch informelle Pflichten. So sind die Bürger*innen eines Staates dazu aufgefordert, sich ausführlich über das politische Geschehen und über wichtige Sachverhalte zu informieren, um sich auf diese Weise adäquat am politischen Prozess beteiligen zu können. Und auch in Staaten, in denen keine Wahlpflicht besteht, sind Bürger*innen, die verkünden, sich nicht an den Wahlen zu beteiligen, oftmals einem gewissen sozialen Druck ausgesetzt, sich eben doch zu beteiligen.

Dies ist eine Aufzählung von Bürden, die mit der Staatsbürgerschaft einhergehen. Man kann auf dieser Grundlage, wie das Ypi und de Schutter (2015) tun, zugunsten einer verpflichtenden Staatsbürgerschaft für diejenigen Migrant*innen argumentieren, die längerfristig im Land leben. Sie behaupten, dass eine verpflichtende Staatsbürgerschaft deshalb gerechtfertigt sei, weil sonst die Bürden ungleich verteilt sind: Diejenigen, die keine Staatsbürgerschaft haben, müssen diese Bürden nicht tragen,

\footnotetext{
${ }^{6}$ Klarerweise ist hier und im Folgenden von demokratischen Staaten die Rede.

7 In diesem Punkt sind sich auch Kommunitaristen wie Walzer und Liberale wie Carens einig; siehe Walzer (2006: 98-105) und Carens (2005: 35-41).

8 Zur Diskussion darüber, wie genau dieses Prinzip zu verstehen ist, siehe etwa Goppel (2012).

9 Allerdings sind noch nie entsprechende Strafverfahren eingeleitet worden (vgl. Robbe und Kuhn 2009).
} 
obwohl auch sie gleichermaßen betroffen sind von den Entscheidungen des Staates und auch sie auf anderen Wegen, etwa durch wirtschaftliche Betätigung, Einfluss ausüben auf andere Staatsbürger*innen. Letztendlich ist es also unfair, wenn nur ein Teil derjenigen, die in einem Staat leben und von seinen Entscheidungen betroffen sind, die Bürden der Mitbestimmung tragen. Ebendiese gerechtigkeitsbasierte Überlegung kann auch angeführt werden, um zu begründen, warum Migrant*innen sich am demokratischen Entscheidungsfindungsprozess beteiligen sollen.

Zum Schluss, dass alle diejenigen, die in einer Demokratie leben, sich an den demokratischen Prozessen beteiligen sollten, kann man auch unter Hinweis auf demokratietheoretische Überlegungen kommen - und diese Überlegungen können ebenfalls als Begründung für die gerade genannten informellen Pflichten von Staatsbürger*innen gelten. Mill etwa sieht die Vorteile der Demokratie zum einen darin, dass sie es am besten gewährleiste, dass die Interessen der Einzelnen vertreten werden, weil hier jeder selbst seine bzw. ihre Interessen vertreten kann, und jede*r selbst diese Interessen am besten kennt. Zudem trägt ihm zufolge die Beteiligung am demokratischen Entscheidungsfindungsprozess zur intellektuellen Bildung sowie zur gegenseitigen Verständigung bei. Die Auseinandersetzung mit politischen Fragen zwinge einen dazu, sich zu informieren und sich mit der Perspektive anderer auseinanderzusetzen und also dazu, die oft allzu enge eigene Sichtweise auf Dinge zu hinterfragen. Auf diese Weise lerne man, sich nicht nur von seinen eigenen Neigungen leiten zu lassen, sondern von Grundsätzen, die sich aus dem Gemeinwohl ergeben. Die Demokratie, das geht aus Mills Aufzählung der Vorzüge des aktiven Charakters hervor, funktioniert demnach nur dann, wenn die Bürger*innen sich auch tatsächlich beteiligen (Mill 1971: 59-76). Eine ähnliche Überlegung ergibt sich aus der Idee, dass die Demokratie verstanden werden kann als Regierungsform, in der die Rechtfertigung eine zentrale Rolle spielt. Wer auch immer in einem Staat lebt und Entscheidungen trifft, die andere (in relevanter Weise) betreffen, sollte demzufolge bereit sein, diese Entscheidungen öffentlich zu rechtfertigen. Genau dies aber kann durch Teilnahme am politischen Entscheidungsprozess erreicht werden (Stout 2004: 6). Die beiden hier nur äußerst knapp skizzierten demokratietheoretischen Überlegungen sind Argumente dafür, dass sich alle diejenigen, die den politischen Entscheidungen eines Staates unterworfen sind, auch an der politischen Willensbildung und Entscheidungsfindung beteiligen sollten.

Diese Überlegungen zeigen, dass es gute Argumente für die Forderung gibt, dass sich Migrant*innen politisch einbringen sollen, sei es aus Gründen der Gerechtigkeit und/oder aus den genannten demokratietheoretischen Überlegungen. Sie sprechen freilich auch dafür, dass der Staat den längerfristig im Land lebenden Migrant*innen die politische Partizipation ermöglichen sollte, vielleicht sogar dafür, die Staatsbürgerschaft für sie verpflichtend zu machen. Im Rahmen des politischen Liberalismus kann man also für eine Forderung nach politischer Partizipation argumentieren. Kann man, ausgehend von diesen Überlegungen, auch zugunsten einer Forderung nach Integration in weiteren Dimensionen argumentieren? 


\section{Voraussetzungen der politischen Partizipation}

Ein Gedanke, der das nahelegt, lautet, dass die Fähigkeit zur politischen Partizipation in einem konkreten Staat auf bestimmten Voraussetzungen beruht. Wer sich in den politischen Entscheidungsprozess einbringt, sollte sich adäquat informieren über das, worüber entschieden werden soll, sollte in den Dialog treten mit anderen. Darüber hinaus sollte man auch gewisse Werte schätzen und gewisse Einstellungen und Haltungen einnehmen - zumindest im Bereich der geteilten politischen Kultur -, die einer Demokratie angemessen sind. Wenn das zutrifft, dann kann auch auf Grundlage einer Forderung nach politischer Integration zugunsten einer Forderung nach zumindest eines gewissen Maßes an kultureller, sozialer und identifikativer Integration argumentiert werden. Ich werde diese Punkte im Folgenden etwas ausführlicher erläutern.

Viele politische Entscheidungen eines Staates beziehen sich entweder direkt auf partikulare kulturelle Gepflogenheiten, Sitten, Konventionen eines Staates, oder sind zumindest indirekt mit diesen und vor allem auch mit der partikularen Geschichte des Staates verbunden. Zu den Themen, für die das sicherlich zutrifft, gehören z.B. die Fragen nach der staatlichen Förderung von Kulturveranstaltungen, nach der Legitimität von Schwangerschaftsabbrüchen oder nach einem Tempolimit auf Autobahnen. Ganz egal, wie man zu diesen Fragen steht; wenn man sie diskutieren möchte, dann ist es unerlässlich, etwas über die Genealogie der entsprechenden Gesetze zu wissen, und also etwas darüber zu wissen, warum bestimmte Regelungen in einem Staat große Zustimmung erfahren bzw. auf große Ablehnung stoßen. Viele derartiger Regelungen lassen sich erklären durch Hinweis auf bestimmte Gepflogenheiten, auf eine im Staat wichtige Religion, auf im Staat weit geteilte moralische Ansichten oder auf zentrale Wirtschaftszweige des Staates. Bei den entsprechenden Gesetzen handelt es sich klarerweise um Artefakte, in die auch ihre jeweilige Entstehungsgeschichte samt ihrer Machtstrukturen eingeschrieben ist. Genuin politisches Denken beruht gerade darauf, auch diese Aspekte zu berücksichtigen (Geuss 2008).

Politische Partizipation erfordert die Auseinandersetzung mit derartigen Fragen, und deshalb erfordert sie es auch, sich zu informieren über die entsprechenden Hintergründe. Da diese Hintergründe oftmals in den partikularen kulturellen Gepflogenheiten, Sitten, Konventionen und der Geschichte eines Staates liegen, ist es demnach gefordert, diese zu kennen, sich mit diesen auseinanderzusetzen. Dies ist sicherlich keine Anpassung an „die“ Kultur eines Staates, aber nichtsdestotrotz ein Teil der kulturellen Integration, nämlich der Erwerb von funktionalem Wissen, der es ermöglicht, sich einzubringen (Heckmann 2015: 160).

Darüber hinaus erfordert demokratische Partizipation auch das Gespräch, die Diskussion. Politische Entscheidungen betreffen typischerweise sehr viele Menschen und sollten deshalb diskutiert werden mit denjenigen, die davon (in relevanter Weise) betroffen sind. Eine Voraussetzung dafür ist das Beherrschen der bzw. einer der Landessprachen. Es handelt sich dabei nicht im strikten Sinn um eine notwendige Voraussetzung, denn gelingende Kommunikation setzt nicht in jedem Fall eine geteilte Landessprache voraus. Politische Fragen lassen sich auch auf anderem Wege diskutieren: Wenn es um das direkte Gespräch geht etwa in einer geteilten dritten Sprache, die nicht die oder eine der Landessprachen ist. Möglich ist es auch, dass 
Medien - in Form von Zeitungen, Magazinen, dem Fernsehen usf. - vermitteln zwischen den Menschen, und damit ebenfalls eine tatsächlich geteilte Sprache ersetzen. Allerdings wird es immer wieder Situation geben, in denen derartige Hilfsmittel nicht vorhanden sind, so dass es durchaus gerechtfertigt ist, darauf zu bestehen, dass Migrant*innen die bzw. eine der Landessprachen beherrschen. Das Beherrschen der bzw. einer der Landessprachen stellt einen weiteren Aspekt der kulturellen Integration dar. ${ }^{10}$

Darüber hinaus ist die Diskussion mit den von politischen Entscheidungen betroffenen auch ein erster Schritt der sozialen Integration: Demokrat*innen sollten miteinander diskutieren - Migrant*innen mit den Einheimischen und genauso natürlich andersherum -, und das setzt eine gewisse soziale Integration, also die Bekanntschaft von Migrant*innen mit Einheimischen voraus.

Zuletzt stellt die politische Partizipation auch einen Aspekt der identifikativen Integration dar. Demokratie ist ein kollektives Projekt, bei dem die einzelnen Teilnehmer*innen in vielerlei Weise aufeinander angewiesen sind. Sie sind es, weil sie miteinander diskutieren müssen, um zu gerechtfertigten Entscheidungen gelangen zu können, und natürlich auch, weil sie dann miteinander handeln müssen, um Entscheidungen umzusetzen. Solch kollektives Entscheiden und Handeln funktioniert nur, wenn man sich als Teil eines gemeinsamen Projektes begreift und sich dementsprechend mit ihm identifiziert. ${ }^{11}$ Dies aber bedeutet eben auch die Identifikation mit einem partikularen demokratischen Projekt - etwa der Demokratie in Deutschland, Belgien oder Frankreich. Eine solche Identifikation schließt nicht ein, dass man alle Aspekte des demokratischen Prozesses in einem Staat gutheißen muss, oder dass man nicht einzelne Aspekte kritisieren darf. Aber sie verlangt doch eine Zustimmung zu grundlegenden Prinzipien und Ideen, die der Demokratie zugrunde liegen und die Bereitschaft, Änderungsvorschläge zu unterbreiten, wenn man mit der partikularen Interpretation des demokratischen Prozederes in einem Staat nicht einverstanden ist. ${ }^{12}$

\footnotetext{
10 Das heißt nicht, dass Staaten sich nicht darum bemühen sollten, neu ankommenden Migrant*innen bestimmte Informationen in ihnen verständlichen Sprachen zugänglich zu machen; und auch nicht, dass es keinen Unterricht in Muttersprachen von Migrant*innen geben darf (siehe dazu Kymlicka (1995: 97), der auf Untersuchungen hinweist, denen zufolge muttersprachlicher Unterricht oftmals dazu führt, dass auch die Landessprache besser gesprochen wird). Aber das langfristige Ziel sollte dennoch darin bestehen, dass die Migrant*innen die bzw. einer der Landessprachen beherrschen.

11 Diese Ansicht beruht auf einer Konzeption der Demokratie, die in etwa der entspricht, die Taylor (2002) als drittes Demokratiemodell vorstellt. Dieses steht zwischen dem ökonomischen - dem zufolge die Demokratie nur ein Mittel ist, die konkurrierenden Zielsetzungen der Mitglieder friedlich und wirksam durchzusetzen - und dem rousseau'schen Modell, das einen Gemeinwillen voraussetzt. Taylors, von de Tocqueville und Arendt inspiriertes drittes Modell steht zwischen diesen beiden, indem es Platz lässt für konkurrierende Zielsetzungen der Bürgerinnen und Bürger, aber zugleich daran festhält, dass es eines „Identifikationspols“ bedarf, der im Gesetz ,als dem Inbegriff der zentralen Institutionen und Verfahren des politischen Systems“ besteht (Taylor 2002: $19 \mathrm{ff}$ ). Diese Idee steht der des „Verfassungspatriotismus“ sehr nahe; siehe dazu etwa Müller (2010).

12 Auch der Verfassungspatriotismus ist laut Müller (2010: 74f) ,primär als ein Projekt zu verstehen, und nicht als eine Art invariante Loyalitätsleistung, welche sich auf ein feststehendes Objekt bezieht [...]“. Dabei dürfen die Bürgerinnen und Bürger dennoch nicht ,in einem Zustand permanenter Ambivalenz gegenüber den Verfassungskernpunkten leben oder diese als rein provisorisch betrachten“.
} 
Zuletzt ist die Demokratie eine Regierungsform, die nur funktionieren kann, wenn die Teilnehmenden gewisse Werte, Einstellungen und Haltungen teilen. Da die Diskussion das zentrale Element der demokratischen Entscheidungsfindung ist, sind dies Werte, Einstellungen und Haltungen, die (möglichst produktive) Diskussionen ermöglichen: Demokrat*innen sollten offen sein für die Meinungen ihrer Diskussionspartner*innen; sie sollten willig und fähig sein, ihre eigenen Meinungen zu hinterfragen; sie sollten rationalen Argumenten zugänglich sein und dabei ihre Emotionen in gewissen Grenzen halten; sie sollten tolerant sein, ihre Diskussionspartner*innen respektieren und ihnen gegenüber ein gewisses Maß an Einfühlungsvermögen mitbringen (Nida-Rümelin 2006: 25). ${ }^{13}$ Die Übernahme von Werten und die Änderung von Einstellungen und Haltungen zählt wiederum zur kulturellen Integration (Heckmann 2015: 159).

Die politische Integration beruht demnach auf Voraussetzungen, die als Aspekte von Integration in den genannten Dimensionen verstanden werden können. Dabei werden allerdings nur ausgesuchte Aspekte der Integration in der jeweiligen Dimension berührt: Sowohl die kulturelle als auch die soziale und die identifikative Dimension umfassen z.B. bei Heckmann (2015) noch weitere Aspekte als die hier angeführten, die jeweils in einem Bezug zur politischen Integration stehen. Es bleiben also im Rahmen der hier vorgelegten Überlegungen einige Aspekte der Integration unberücksichtigt, so z.B. auch die sicherlich ebenfalls zentrale Integration in den Arbeitsmarkt bzw. die Wirtschaft. Nichtsdestotrotz kann man festhalten, dass, wenn die Forderung nach politischer Partizipation begründet ist, man dann auch eine Forderung begründen kann, dass Migrant*innen sich in den eben genannten Weisen kulturell, sozial und auch identifikativ integrieren sollen.

Natürlich gilt gleichermaßen, dass der Staat eine entsprechende Pflicht hat, Migrant*innen bei der Integration zu unterstützen: Er soll z.B. leicht zugängliche und gut verständliche Informationen zu historischen Ereignissen und zu kulturellen Gepflogenheiten bereitstellen, die wichtig sind für das Verständnis politischer Entscheidungen; darüber hinaus auch Informationen zum partikularen politischen System; er soll Sprachkurse anbieten und Orte und Anlässe schaffen, an denen Einheimische und Migrant*innen miteinander ins Gespräch kommen können.

Da die Forderung nach Integration sowohl Gerechtigkeits- als auch demokratietheoretischen Überlegungen entspringt gilt, dass sie sich ebenso an die Einheimischen richtet. Insofern diese gut Bescheid wissen über die kulturellen Gepflogenheiten, Sitten, Konventionen des Staates, in dem sie leben, sind sie von einigen der genannten Forderungen weniger stark angesprochen als Migrant*innen. Gerade

\footnotetext{
13 Auch Larmore (1993: 150) hält fest, dass der politische Liberalismus eine „moralische Konzeption“ ist, die auf den Normen des gegenseitigen Respekts und des rationalen Dialogs beruht. Mit der Behauptung, dass sich durchaus eine Forderung begründen lässt, bestimmte Werte zu übernehmen, widerspreche ich Özmen (2015: 358), der zufolge eine Ethik der Integration nichts mit der Anerkennung oder Internalisierung von Werten zu tun habe. Einige der in Abschnitt 3 zitierten Forderungen von de Maizière - etwa die nach respektvollem Verhalten und Toleranz - lassen sich also durchaus im Rahmen des Liberalismus rechtfertigen. Die Begründung lautet dann nicht, dass dies (vermeintliche) „Lebensgewohnheiten“ der Deutschen seien, an die sich Migrant*innen anzupassen hätten, sondern, dass diese Einstellungen eine Voraussetzung für das demokratische Zusammenleben darstellen.
} 
die Forderung nach Übernahme demokratieförderlicher Werte, Einstellungen und Haltungen richtet sich aber in der gleichen Vehemenz an die Einheimischen. ${ }^{14}$

\section{Ein möglicher Einwand}

Die Ausführungen bis hierhin sollen belegen, dass der Staat das Recht hat, von Migrant*innen (sowie von den Einheimischen) $\mathrm{zu}$ fordern, gewisse Handlungen auszuführen und Einstellungen zu übernehmen, welche die Integration in den eben genannten Dimensionen befördern. Ein möglicher Einwand gegen die hier vorgestellte Argumentation ist allerdings noch nicht genannt worden. Dieser Einwand beruht auf einer bestimmten Interpretation des Liberalismus und besteht in der Behauptung, dass die zentrale Tugend des Liberalismus die Toleranz gegenüber abweichenden Meinungen und Lebensweisen sei und es deshalb kein Ziel eines liberalen Staates sein könne, auf die Konformität der Meinungen seiner Bürger*innen hinzuwirken. Aus diesem Grund sei es liberalen Staaten nicht gestattet, ihre Bürger*innen zu liberalen Werten und zur Demokratie zu erziehen (Kukathas 2001). Wenn das zutrifft, dann ist die hier vorgestellte Begründung einer Forderung nach Integration auf Grundlage des politischen Liberalismus nichtig.

Zunächst einmal ist festzuhalten, dass tatsächlich gewisse Spannungen innerhalb des politischen Liberalismus bestehen, die man folgendermaßen beschreiben kann: Einerseits soll der Staat dem politischen Liberalismus zufolge seinen Bürger*innen möglichst viele Freiheiten lassen. $\mathrm{Zu}$ diesen Freiheiten gehören etwa die, selbst zu bestimmen, welcher Religion man angehören will, welche Küche man bevorzugt - und es scheint zumindest prima facie sehr plausibel zu sein, dass zu diesen Freiheiten auch die gehört, selbst zu entscheiden, ob man sich am demokratischen Entscheidungsfindungsprozess aktiv beteiligen will oder nicht. Andererseits bedarf die Demokratie der aktiven Teilnahme der Bürger*innen. Eine parallele Überlegung gilt in Bezug auf die Einstellungen und Haltungen der Bürger*innen eines liberalen Staates. Einerseits sollte der liberale Staat diese nicht beeinflussen, da ein solcher Eingriff in die Überzeugungen die Gewissensfreiheit beschränkt; andererseits muss zwischen den Bürger*innen eines liberalen Staates ein gewisser Grundkonsens herrschen, der diesen liberalen Staat ermöglicht - dazu gehört etwa die Haltung, sich gegenseitig als Gleiche anzuerkennen. ${ }^{15}$

Die Frage lautet also an dieser Stelle, in welche Richtung man diese Spannungen auflöst. Viele liberale Staaten setzen auf die Erziehung ihrer Bürger*innen. Dies geschieht vor allem in der Schule, aber auch durch Programme, Veranstaltungen usf., die sich an Erwachsene richten. Hierbei werden zum einen Informationen übermittelt, die wichtig sind, um politische Entscheidungen treffen zu können und

\footnotetext{
14 Das betont auch Carens (2015: 64). Untersuchungen zur klassenspezifischen Wahlbeteiligung belegen außerdem, dass die demokratische Teilhabe vor allem durch strukturelle ökonomische und soziale Faktoren bestimmt wird, und nicht dadurch, dass jemand Migrant*in ist oder nicht. Siehe dazu etwa Bödeker (2012) und Schäfer (2015).

15 Es sind diese Spannungen, die dem sogenannten Böckenförde-Diktum zugrunde liegen; siehe Böckenförde (1991).
} 
es wird das partikulare politische System erklärt. Durch diese Bemühungen sollen sich bei den Bürger*innen außerdem Werte wie Toleranz, Respekt und Offenheit verbreiten, sie sollen lernen, sich gegenseitig als Gleiche anzuerkennen und vernünftig miteinander zu diskutieren. Auf welche Argumente kann man sich dabei berufen? Das wohl stärkste Argument lautet, dass die Fortexistenz des liberalen Staates darauf angewiesen ist, dass die Bürger*innen gewisse Haltungen haben und bestimmte Verhaltensweisen an den Tag legen (Macedo 1995: 225). Demzufolge hat der liberale Staat das Recht, seine Bürger*innen zur Annahme dieser Haltungen und Verhaltensweisen zu erziehen. Das sollten auch diejenigen anerkennen, für die die Toleranz gegenüber abweichenden Meinungen und Lebensweisen die zentrale liberale Tugend ist, denn diese Toleranz ist ebenfalls in Gefahr, sollte der liberale Staat nicht fortbestehen.

Allerdings überzeugt dieses Argument nicht alle. Kukathas (2001: $326 \mathrm{ff}$.) bringt zwei Einwände dagegen vor. Erstens funktioniere eine solche staatliche Erziehung nicht, und zweitens bedürfe es keiner staatlichen Erziehung dafür, dass es Menschen gebe, die die liberale Tugend der Toleranz lebten.

Dafür, dass eine solche Erziehung nicht funktionieren könne argumentiert Kukathas unter Berufung auf Rousseau, und zwar in der Interpretation von Judith Shklar. Shklars Rousseau zufolge kann die staatliche Erziehung bewirken, dass die Bürger*innen die gewünschten Verhaltensweisen zeigen - das allerdings nur, solange sie unter staatlicher Beobachtung stehen (Kukathas 2001: 328; Shklar 1985: 160). Allerdings trägt diese Begründung nicht sehr weit. Dies zum einen deshalb, weil keineswegs klar ist, dass Rousseaus Behauptung durch empirische Befunde gestützt wird. So zeigten etwa die Re-Education-Programme der Vereinigten Staaten in der Bundesrepublik Deutschland durchaus einige Wirkung, die über bloße Lippenbekenntnisse hinausgehen. ${ }^{16}$ Dem Einwand Kukathas' kann man aber auch auf andere Weise begegnen: Wenn es stimmt, dass die staatliche Erziehung wirkungslos ist, dann gelingt es ihr nicht, einen Einfluss auf die Haltungen und Überzeugungen der Bürger*innen auszuwirken. In diesem Fall ist aber auch nicht zu sehen, wieso man in ihr eine unangemessen übergriffige Maßnahme des Staates erblicken sollte. Kukathas' erster Einwand kann also nicht überzeugen.

Kukathas' zweiter Einwand lautet, dass die Anhänger*innen einer staatlichen Erziehung fälschlicherweise davon ausgingen, dass es der Erziehung bedürfe, damit Menschen demokratische Überzeugungen und Haltungen entwickelten, die einer liberalen Demokratie angemessen sind. Dem sei aber nicht so; vielmehr würden diese Überzeugungen und Haltungen natürlich entstehen: „Liberal citizens do come into existence ,naturally“, just as morality does“ (Kukathas 2001: 329). Das zeige sich daran, dass auch in illiberalen Gesellschaften immer wieder Menschen mit liberalen Einstellungen und Überzeugungen vorkämen.

Auch dieser Einwand kann nicht überzeugen. Zwar trifft es wohl zu, dass immer wieder auch Menschen, die nicht entsprechend erzogen wurden, ,von sich aus“ liberale Einstellungen und Überzeugungen entwickeln. Wenn dem nicht so wäre, dann hätte man Probleme, zu erklären, wie liberale - oder überhaupt neue - Einstellungen und Überzeugungen entstehen können. Allerdings ist dieses Zugeständnis

16 Zur Diskussion siehe etwa einige der Beiträge in Gerund und Paul (2015) sowie Roth (2005: 25-52). 
kein ausreichender Grund für die Behauptung, dass liberale Staaten auf die Erziehung ihrer Bürger*innen vollständig verzichten könnten. Dies deshalb, weil liberale Staaten eben nicht nur einiger Bürger*innen bedürfen, die liberale Einstellungen und Überzeugungen vertreten - und es einfach ein zu großes Risiko ist, dies dem Zufall bzw. den Zeitläuften zu überlassen. Schon ein kurzer Blick in die Geschichte offenbart, wie schwer es war, liberale Ideen durchzusetzen und wie viele Widerstände dabei überwunden werden mussten. In Anerkennung dieses Tatbestandes wäre es sehr leichtsinnig, den Fortbestand eines liberalen Staates aufs Spiel zu setzen aufgrund des Gedankens, dass ein solcher ja immer wieder entstehen könne. Doch Kukathas weist auch diesen Gedankengang zurück mit der Begründung, dass die Fortexistenz liberaler Staaten gar nicht darauf beruhe, dass alle oder die Mehrheit der Bürger*innen liberale Einstellungen haben:

What is necessary to sustain a liberal political order is not institutions geared up to inculcate liberal virtues or liberal norms of citizenship but the liberal practice of toleration of dissent. Moreover, while it might be useful to have a number among the citizenry who are strongly committed to the principles of liberalism, it is not clear that all, or even a plurality of citizens need to be so committed. Liberal societies seem well capable of operating with a good deal of dissenting opinion, and a great deal of political apathy. Too great a level of indifference to matters of principle might be dangerous since it is important that there be enough interest among the people to object to a tyrannical or dishonest government. But it is not clear that a liberal society cannot get by perfectly well with much of the population taking no interest in politics whatsoever. (Kukathas 2001: 329)

Nun stimmt es sicherlich, dass die Fortexistenz eines liberalen Staates nicht darauf beruht, dass alle Bürger*innen liberale Einstellungen haben. Allerdings zeigen doch gerade die jüngsten politischen Entwicklungen - etwa in den USA, in Ungarn, Polen und der Türkei -, wie groß die Gefahr ist, die von illiberalen politischen Bewegungen bzw. Regierungen innerhalb liberaler Staaten ausgeht. Diese Entwicklungen machen deutlich, wie sehr die Demokratie auf Demokrat*innen angewiesen ist. Die „bloßen“ Gesetze und Institutionen können, auch dann, wenn sie eigentlich demokratische Grundsätze verkörpern, allzu oft in einer Weise interpretiert bzw. benutzt (oder einfach übergangen) werden, die sie zu Werkzeugen antidemokratischer und antiliberaler Machenschaften verkommen lässt. Das alles lässt m.E. nur den Schluss $\mathrm{zu}$, dass der Fortbestand der liberalen Demokratie keinesfalls gesichert ist, sondern einer andauernden Verteidigung gegen ihre Verächter*innen bedarf. Die Erziehung der Bürger*innen zu demokratischen, liberalen Werten ist darum ein legitimer Bestandteil eines demokratischen, liberalen Staates. ${ }^{17}$ Wenn aber der liberale Staat von seinen Bürger*innen fordern darf, dass sie bestimmte Einstellungen und Überzeugungen annehmen, dann darf er dasselbe auch von Migrant*innen verlangen - die ja in vielen Fällen zukünftige Staatsbürger*innen sein werden. ${ }^{18}$ Folglich ist die

17 Das sehen außer Macedo (1995, 2000) auch Barry (2001: 199-237) und Gutmann (1987) so.

18 Ähnlich argumentiert Miller (2016: 137). 
Forderung danach, dass sich Migrant*innen in den oben genannten Hinsichten um Integration bemühen sollen, gerechtfertigt.

\section{Fazit}

Ich bin in diesem Aufsatz der Frage nachgegangen, ob der Staat von Migrant*innen fordern darf, sich zu integrieren. Ich habe gezeigt, dass sich auf Grundlage des politischen Liberalismus zugunsten einer solchen Forderung argumentieren lässt. Zur Begründung kann man sich auf Gerechtigkeitsüberlegungen und demokratietheoretische Überlegungen stützen. Es handelt sich also bei der Forderung also nicht um eine Forderung danach, dass sich Migrant*innen einer (vermeintlich) geteilten Kultur oder Lebensform in einem Staat anpassen sollen, obwohl sie durchaus auch beinhaltet, sich in einem gewissen Maße um kulturelle, soziale und auch identifikative Integration zu bemühen. Diese Forderung ergibt sich demnach nicht aus dem Wert der Bewahrung einer partikularen Kultur oder Lebensform, sondern aus einem universellen Ideal der Demokratie. Diesem universellen Ideal kann man unter der Bedingung, dass es (vor allem) die Nationalstaaten sind, die heutzutage politische Mitbestimmung ermöglichen, eben nur folgen, indem man sich auf die partikulare politische Kultur eines bestimmten Staates einlässt, was ein gewisses Maß an kultureller, sozialer und identifikativer Integration in einem Staat erfordert.

Es bleibt außerdem festzuhalten, dass die hier begründete Forderung nach Integration nur einen Teil der Aspekte umfasst, die zur Integration gezählt werden. So kann z.B. eine ebenfalls enorm wichtige Dimension der strukturellen Integration, nämlich die in den Arbeitsmarkt, wohl nicht ohne weiteres als Voraussetzung der politischen Partizipation gelten und darum unter Berufung auf den hier vorgestellten Gedankengang nicht begründet werden. ${ }^{19}$ Die hier begründete Forderung nach Integration ist also begrenzter als die oftmals im öffentlichen Diskurs genannten Forderungen.

Darüber hinaus bin ich im Aufsatz nicht auf die konkrete Umsetzung dieser Forderung eingegangen. M.E. kann die hier vorgestellte Begründung z.B. als Rechtfertigung einer gängigen Praxis dienen, diese Forderung gegenüber Migrant*innen durchzusetzen, nämlich der Verwendung sogenannter Einbürgerungstests, in denen Wissen über die Geschichte eines Staates, das politische System und auch über kulturelle Aspekte abgefragt wird. Gemäß dem hier vorgestellten Argument muss dieses Wissen eine Voraussetzung für die politische Partizipation sein. Man muss allerdings damit rechnen, dass hier Meinungsverschiedenheiten darüber bestehen werden, worin genau denn diese Voraussetzungen bestehen, die ich in Abschnitt 5 nur recht abstrakt charakterisiert habe: Was muss man über das Christentum wissen, um die Bedeutung der religiös motivierten Feiertage der Bundesrepublik Deutschland

19 Den Zusammenhängen zwischen den verschiedenen Dimensionen der Integration geht z.B. Esser (2001: 16f.) nach. 
einschätzen zu können? Was über den Nationalsozialismus, um die deutsche $\mathrm{Au}-$ ßenpolitik verstehen zu können? Über diese Detailfragen muss diskutiert werden. ${ }^{20}$

Zuletzt sei darauf hingewiesen, dass die hier begründete Forderung freilich nicht impliziert, dass Migrant*innen keine Kritik am politischen System des Staates üben dürfen. Vielmehr sind sie, ebenso wie die Einheimischen, aufgefordert, dies zu tun - zumindest, solange sie dabei nicht den Boden demokratischer Vorstellungen verlassen. Eine gelingende Integration bedeutet demnach nicht zwangsläufig eine Abnahme von Konflikten. ${ }^{21}$ Die Integration von Migrant*innen (genau wie die der Einheimischen) in den demokratischen politischen Prozess kann es ihnen ermöglichen, Positionen deutlicher und (öffentlichkeits-)wirksamer zu formulieren als zuvor, was zu einer Zunahme von Konflikten führen kann, die aber zugleich durch den demokratischen Prozess gebändigt werden. ${ }^{22}$

Funding Open Access funding enabled and organized by Projekt DEAL.

Open Access Dieser Artikel wird unter der Creative Commons Namensnennung 4.0 International Lizenz veröffentlicht, welche die Nutzung, Vervielfältigung, Bearbeitung, Verbreitung und Wiedergabe in jeglichem Medium und Format erlaubt, sofern Sie den/die ursprünglichen Autor(en) und die Quelle ordnungsgemäß nennen, einen Link zur Creative Commons Lizenz beifügen und angeben, ob Änderungen vorgenommen wurden.

Die in diesem Artikel enthaltenen Bilder und sonstiges Drittmaterial unterliegen ebenfalls der genannten Creative Commons Lizenz, sofern sich aus der Abbildungslegende nichts anderes ergibt. Sofern das betreffende Material nicht unter der genannten Creative Commons Lizenz steht und die betreffende Handlung nicht nach gesetzlichen Vorschriften erlaubt ist, ist für die oben aufgeführten Weiterverwendungen des Materials die Einwilligung des jeweiligen Rechteinhabers einzuholen.

Weitere Details zur Lizenz entnehmen Sie bitte der Lizenzinformation auf http://creativecommons.org/ licenses/by/4.0/deed.de.

\section{Literatur}

Barry, Brian. 2001. Culture and Equality. An Egalitarian Critique of Multiculturalism. Cambridge, Mass.: Harvard University Press.

Bedorf, Thomas, und Steffen Herrmann. 2016. Das Gewebe des Sozialen. Geschichte und Gegenwart des sozialen Bands. In Das soziale Band. Geschichte und Gegenwart eines sozialtheoretischen Grundbegriffs, Hrsg. Thomas Bedorf und Steffen Hermann, 11-50. Frankfurt a.M.: Suhrkamp.

Böckenförde, Ernst-Wolfgang. 1991. Die Entstehung des Staates als Vorgang der Säkularisation. In Recht, Staat, Freiheit. Hrsg. Ernst-Wolfgang Böckenförde, 92-114. Frankfurt a.M.: Suhrkamp.

Bödeker, Sebastian. 2012. Soziale Ungleichheit und politische Partizipation in Deutschland. Grenzen politischer Gleichheit in der Bürgergesellschaft. https://www.otto-brenner-stiftung.de/fileadmin/user_ data/stiftung/02_Wissenschaftsportal/03_Publikationen/AP01_SozialeUngleichheit_Boedeker_2012_ 02_07.pdf. Zugegriffen: 29. September 2021.

\footnotetext{
20 Außerdem dürfen solche Tests nicht als Vehikel der Diskriminierung bestimmter Religionen bzw. Ethnien dienen; siehe dazu Joppke (2007).

21 Auch Heitmeyer (1997b: 26) weist darauf hin, dass ,,die Gleichsetzung von Integration und Konfliktfreiheit" fehlgeht. In Bezug auf die Integration von Migrant*innen thematisiert diesen Befund El-Mafalaani (2018).

${ }^{22}$ Ich danke den anonymen Gutachter*innen für viele Hinweise und konkrete Verbesserungsvorschläge für meinen Aufsatz.
} 
Carens, Joseph. 1987. Aliens and Citizens: The Case for Open Borders. The Review of Politics 49 (2): 251273.

Carens, Joseph. 2005. The Integration of Immigrants. Journal of Moral Philosophy, 2 (1): 29-46.

Carens, Joseph. 2015. The Ethics of Immigration. Oxford: Oxford University Press.

Cassee, Andreas. 2016. Globale Bewegungsfreiheit. Ein philosophisches Plädoyer für offene Grenzen. Frankfurt a.M.: Suhrkamp.

de Maizière, Thomas. 2017. Wir sind nicht Burka. Bild am Sonntag vom 30.04.2017.

Dietrich, Frank. Hrsg. 2017. Ethik der Migration. Philosophische Schlüsseltexte. Frankfurt a.M.: Suhrkamp.

El-Mafalaani, Aladin. 2018. Das Integrationsparadox: Warum gelungene Integration zu mehr Konflikten führt. Köln: Kiepenheuer \& Witsch.

Esser, Hartmut. 2000. Soziologie. Spezielle Grundlagen. Band 2: Die Konstruktion der Gesellschaft. Frankfurt a.M. u.a.: Campus.

Esser, Hartmut. 2001. Integration und ethnische Schichtung. http://www.mzes.uni-mannheim.de/publications/ wp/wp-40.pdf. Zugegriffen: 29.09.2021.

Freiman, Christopher und Javier Hidalgo. 2016. Liberalism or Immigration Restrictions, but not both. Journal of Ethics and Social Philosophy 10 (2): S. 1-22.

Friedrichs, Jürgen und Wolfgang Jagodzinski. 2008. Theorien sozialer Integration. In Integration - Desintegration. Ein Reader zur Ordnungsproblematik moderner Gesellschaften, Hrsg. Peter Imbusch und Wolfgang Jagodzinski, 93-117. Wiesbaden: VS Verlag für Sozialwissenschaften.

Gerund, Katharina, und Heike Paul. Hrsg. 2015. Die amerikanische Re-Education Politik nach 1945. Interdisziplinäre Perspektiven auf „America's Germany“. Bielefeld: Transcript.

Geuss, Raymond. 2008. Philosophy and Real Politics. Princeton und Oxford: Princeton University Press.

Goppel, Anna. 2012. Wahlrecht für Ausländer? In Migration und Ethik, Hrsg. Andreas Cassee und Anna Goppel, 255-274. Münster: Mentis.

Gutmann, Amy. 1987. Democratic Education. Princeton: Princeton University Press.

Gutmann, Amy. 1993. Die kommunitaristische Kritik des Liberalismus. In Kommunitarismus. Eine Debatte über die moralischen Grundlagen moderner Gesellschaften, Hrsg. Axel Honneth, 68-83. Frankfurt a.M. u.a.: Campus, S. 68-83.

Habermas, Jürgen. 1992. Staatsbürgerschaft und nationale Identiät. In Faktizität und Geltung. Beiträge zur Diskurstheorie des Rechs und des demokratischen Rechtsstaats, Hrsg. Jürgen Habermas, 632-660.. Frankfurt a.M.: Suhrkamp.

Habermas, Jürgen. 2002. Begründete Enthaltsamkeit. Gibt es postmetaphysische Antworten auf die Frage nach dem ,richtigen Leben'? In Die Zukunft der menschlichen Natur. Auf dem Weg zu einer liberalen Eugenik?, Hrsg. Jürgen Habermas, 11-33. Frankfurt a.M.: Surhkamp.

Heckmann, Friedrich. 2015. Integration von Migranten. Einwanderung und Nationenbildung. Wiesbaden: Springer VS.

Heitmeyer, Wilhelm. Hrsg. 1997a. Bundesrepublik Deutschland: auf dem Weg von der Konsens- zur Konfliktgesellschaft. Bd. 2: Was hält die Gesellschaft zusammen? Frankfurt a.M.: Suhrkamp.

Heitmeyer, Wilhelm. 1997b. Gibt es eine Radikalisierung des Integrationsproblems? In Bundesrepublik Deutschland: auf dem Weg von der Konsens- zur Konfliktgesellschaft. Bd. 2: Was hält die Gesellschaft zusammen?, Hrsg. Wilhelm Heitmeyer, 23-65. Frankfurt a.M.: Suhrkamp.

Heitmeyer, Wilhelm. 1997c. Einleitung: Sind individualisierte und ethnisch-kulturell vielfältige Gesellschaften noch integrierbar? In Bundesrepublik Deutschland: auf dem Weg von der Konsens- zur Konfiktgesellschaft. Bd. 2: Was hält die Gesellschaft zusammen?, Hrsg. Wilhelm Heitmeyer, 9-19. Frankfurt a.M.: Suhrkamp.

Hess, Sabine, und Johannes Moser. 2009. Jenseits der Integration. Kulturwissenschaftliche Betrachtungen einer Debatte. In No Integration?! Kulturwissenschaftliche Beiträge zur Integrationsdebatte in Europa, Hrsg. Sabine Hess, Jana Binder und Johannes Moser, 11-25. Bielefeld: Transcript.

Joppke, Christian. 2007. Beyond national models: Civic integration policies for immigrants in Western Europe. West European Politics 30 (1): 1-22.

Köppl, Stefan. Hrsg. 2013. Was hält Gesellschaften zusammen? Ein internationaler Vergleich. Wiesbaden: Springer VS.

Kukathas, Chandras. 2001. Education and Citizenship in Diverse Societies. International Journal of Educational Research. https://doi.org/10.1016/S0883-0355(01)00027-1

Kymlicka, Will. 1990. Contemporary Political Philosophy. An Introduction. Oxford u.a.: Oxford University Press.

Kymlicka, Will. 1995. Multicultural Citizenship. Oxford: Clarendon Press. 
Ladwig, Bernd. 2012. Offene Grenzen als Gebot der Gerechtigkeit? In Migration und Ethik, Hrsg. Andreas Cassee und Anna Goppel, 67-88. Münster: Mentis.

Lammert, Norbert. Hrsg. 2006. Verfassung, Patriotismus, Leitkultur. Was unsere Gesellschaft zusammenhält. Hamburg: Hoffmann und Campe.

Larmore, Charles. 1993. Politischer Liberalismus. In Kommunitarismus. Eine Debatte über die moralischen Grundlagen moderner Gesellschaften, Hrsg. Axel Honneth, 131-156. Frankfurt a.M. u.a.: Campus.

Macedo, Stephen. 1995. Multiculturalism for the Religious Right? Defending Liberal Civic Education. Journal of Philosophy of Education 29 (2): 223-238.

Macedo, Stephen. 2000. Diversity and Distrust. Civic Education in a Multicultural Society. Cambridge, Mass.: Harvard University Press.

Mill, John Stuart. 1971. Betrachtungen über die repräsentative Demokratie. Paderborn: Schöningh.

Miller, David. 2016. Strangers in our Midst. The Political Philosophy of Immigration. Cambridge, MA: Harvard University Press.

Modood, Tariq. 2007. Multiculturalism: A civic Idea. Cambridge, Mass. u.a.: Polity.

Müller, Jan-Werner. 2010. Verfassungspatriotismus. Frankfurt a.M.: Suhrkamp.

Münch, Richard. 1997. Elemente einer Theorie der Integration moderner Gesellschaften. Eine Bestandsaufnahme. In: Bundesrepublik Deutschland: auf dem Weg von der Konsens- zur Konfliktgesellschaft. Bd. 2: Was hält die Gesellschaft zusammen?, Hrsg. Wilhelm Heitmeyer, 66-109. Frankfurt a.M.: Suhrkamp.

Neuhäuser, Christian. 2015. Das Recht auf Einwanderung aus moralstrategischer Perspektive: Ein Plädoyer für eine Ethik der Integration. Zeitschrift für Praktische Philosophie 2 (2): 397-408.

Nida-Rümelin, Julian. 2006. Demokratie und Wahrheit. München: Beck.

Özmen, Elif. 2015. Warum eigentlich Werte? Einige Gedanken zur ,Flüchtlingskrise‘. Zeitschrift für praktische Philosophie 2 (2): 349-360.

Robbe, Patrizia, und Lena Kuhn. 2009. Wahlpflicht. Wissenschaftliche Dienste des Deutschen Bundestages, https:/www.bundestag.de/resource/blob/190722/607099a79d57cdc01c3dc34a96d88469/ wahlpflicht-data.pdf. Zugegriffen: 29. September 2021.

Roth, Maren. 2005. Erziehung zur Demokratie? Amerikanische Demokratisierungshilfe im postsozialistischen Bulgarien. Münster: Waxmann.

Schäfer, Armin. 2015. Der Verlust politischer Gleichheit. Warum die sinkende Wahlbeteiligung der Demokratie schadet. Frankfurt a.M.: Campus.

Shklar, Judith. 1985. Men and Citizens. A Study of Rousseau's social theory. Cambridge: Cambridge University Press.

Stein, Tine. 2008. Gibt es eine multikulturelle Leitkultur als Verfassungspatriotismus? Zur Integrationsdebatte in Deutschland. Leviathan 36: 33-53.

Stout, Jeffrey. 2004. Democracy and Tradition. Princeton: Princeton University Press.

Taylor, Charles. 2002. Wieviel Gemeinschaft braucht die Demokratie? In Wieviel Gemeinschaft braucht die Philosophie? Aufsätze zur politischen Philosophie, Hrsg. Charles Taylor, 11-29. Frankfurt a.M.: Suhrkamp.

Teufel, Erwin. Hrsg. 1996. Was hält die moderne Gesellschaft zusammen? Frankfurt a.M.: Suhrkamp.

Tibi, Bassam. 2001. Leitkultur als Wertkonsens. Bilanz einer missglückten deutschen Debatte. Aus Politik und Zeitgeschichte, Bd. 1-2: 23-26.

Walzer, Michael. 2006. Sphären der Gerechtigkeit. Ein Plädoyer für Pluralität und Gleichheit. Frankfurt a.M. u.a.: Campus.

Wellman, Christopher Heath, und Philipp Cole. 2011. Debating the Ethics of Immigration. Is there e right to exclude? Oxford u.a.: Oxford University Press.

Ypi, Lea, und Helder de Schutter. 2015. Mandatory Citizenship for Immigrants. British Journal of Political Science 45: 235-251. 